\title{
Processing Chain of a Radar Network for Safety Improvement in the Usage of Heavy Machinery
}

\author{
Francisco León-Infante, José-Tomás González-Partida, \\ Rodrigo Blázquez-García and Mateo Burgos-García
}

\begin{abstract}
The use of heavy machinery is one of the main causes of accidents in sites as warehouses or construction. These vehicles have several blind spots that encumber their maneuvering and create a collision-prone environment. To ensure safety in these situations, an early warning system capable of avoiding these accidents is required. An innovative solution consists of the use of a on-board, low-cost, K-band radar network. The complete system is to operate with a very low false alarm rate (FAR) to avoid unnecessary stops and loss of trust by the driver. In order to fulfill this requirement, a processing chain that rejects false detections is implemented. It is also responsible for joining data from all the subsystems of the network.
\end{abstract}

Keywords-Collision avoidance; radar clutter; radar signal processing; radar tracking; position estimation.

\section{INTRODUCTION}

The main problem of big vehicles is the existence of several blind spots in the driver's field of view, which leads to many collisions that not only slow down the work process, but can also cause damage to property or personnel. To avoid this hazard, an early warning system to detect collisionprone targets is essential. Nowadays, several kinds of security systems are used for this purpose, such as cameras or sound alarms. However, their performance is dramatically diminished by hostile environments. Particulate matter obstructs cameras' lenses, and intense noises cloak sound alarms. Therefore an innovative solution is needed. The proposed ground-changing early warning system is an on-board radar network, which is affected neither by heavy sounds nor particulate matter. For this application, the crucial specification is a very low false alarm rate (FAR), without compromising detection capability. The main source of false alarms in this system is the ground undesired reflections or clutter. In situations where this effect is prominent, the radar system will create a non-existent collision target at the distance of the reflection peak, reducing the reliability of the system. In order to avoid these false alarms, signal and data processing are implemented to discriminate real targets from false detections.

\section{A. Sensor Scheme}

The proposed system is to mount radar sensors on the rear bumper of the vehicle, where the majority of blind spots are typically located. This idea is depicted in Fig. 1. Many benefits can be derived from the use of multiple sensors in multi-target surveillance systems, such as position computation and more accurate target state estimation [1]

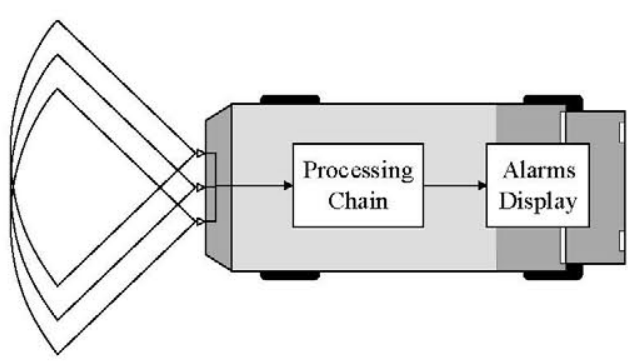

Fig. 1. Proposed radar network aboard the vehicle. The on-board system is located in the critical spot of the machinery (rear bumper). Overlapping beam patterns allow position estimation for more accurate collision alarms.

All radar sensors in the system should have a wide opening angle in azimuth, whereas it is not necessary to have such opening angle in elevation, since all possible targets are expected in a short height range. The sensor beam patterns should overlap to enable position estimation of detected targets by means of two or more range measurements from different locations. This method is called quadratic position-fixing [2]. The proposed sensor scheme consists of tiny low-cost linear frequency modulated continuous wave (LFM-CW) transceivers [3]. The radar waveform allows simultaneous range-velocity resolution, improving detection in multi-target situations.

\section{B. Processing chain architecture}

The processing chain performs two basic tasks: Signal and data processing. It is in the latter where the trickier design specifications are found. Those will be more deeply discussed in section III. Data processing performs target tracking and data fusion. Tracking stage associates consecutive radar observations of the same target into tracks, while data fusion merges these tracks from all the network, allowing estimation of targets' positions. A variety of options are available in terms of architecture for sensor network systems [4]. The proposed alternative, depicted in Fig. 2, is an approach where both sensor-level and central-level tracking are performed. Using this architecture, possible targets are doubly confirmed in order to be the output of the system. This dramatically reduces FAR. As a drawback, a greater delay is introduced by both tracking stages, since persistent plots are needed to produce an alarm. This fact is analogous to target verifying in order to have a very low FAR, although it is still preferable rather than a false detection. To counteract the delay, shorter detection cycles are implemented. 


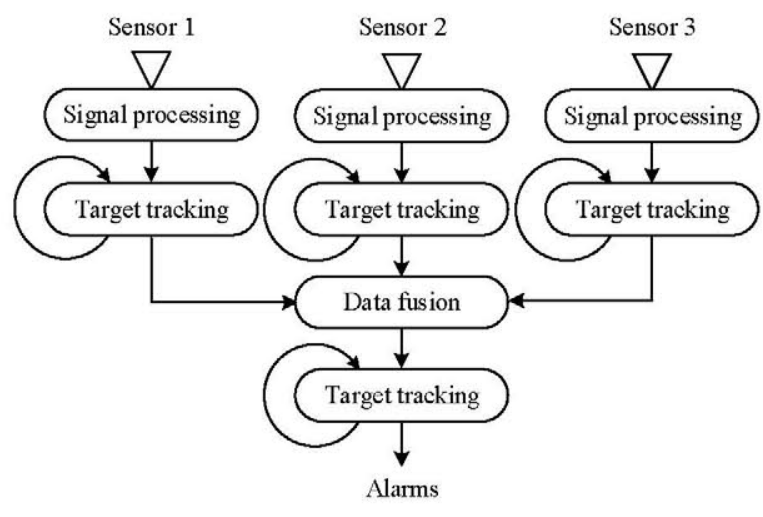

Fig. 2. Two-level-tracking proposed architecture, where possible targets are verified both before and after data fusion, in order to achieve a very-low FAR.

\section{Detection Requeriments}

Due to the application, the system is to operate with approximately one false alarm per workday. This way, the system should detect a person with a probability higher than 0.95 , being the FAR lower than $10^{-1}$. Monte Carlo simulations have been done in order to estimate the detection probability and FAR of a single radar sensor, showing that a standalone sensor cannot meet the requirements, as it has a FAR of about $10^{-4}$ for the needed detection probability [3]. Using a network with target tracking and data fusion, the number of false tracks can be reduced to the required levels.

\section{SIgNAL PROCESSING}

LFM sensors transmit a burst of frequency ramps. Each received ramp is demodulated with a copy of the transmitted ramp, obtaining the beat signal. A fast Fourier transform (FFT) of the received signal performs the pulse compression to get the range profiles. Then, several consecutive range profiles are allocated in a matrix form, and another FFT is carried across all of them. In this way, the Doppler compression is achieved. A range-Doppler matrix (RDM) such as the one depicted in Fig. 3 is obtained. This process gives the ability to evaluate target distance and radial velocity simultaneously by searching in which bins of the RDM are located the local maxima.

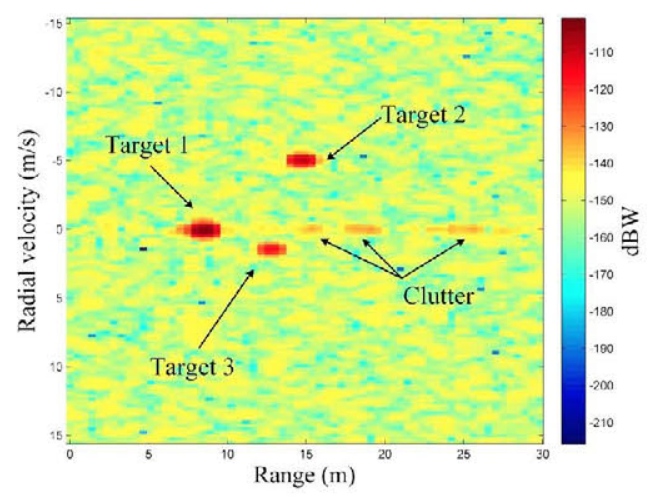

Fig. 3. RDM example, with peaks caused by three targets and undesired reflections. Ground clutter is the line located at zero relative radial velocity. This line spreads along Doppler dimension with increasing range.

\section{A. CFAR application}

In typical environments, the target appears before a background filled with white noise and undesired reflections, adding random power level along the whole spectrum. This fact calls for adaptive signal processing techniques operating with a variable detection threshold, dependent on the neighbor noise level, which allows maintaining a constant FAR (CFAR) in changing situations. In order to obtain the needed local noise and clutter information, a certain environment defined by a reference window around the radar cell under test (CUT) in the RDM must be analyzed. Several CFAR schemes based on a one-dimensional reference window have been published [5] but for our system, it is preferable to use a two-dimensional reference window. This leads to better estimation of local noise and clutter power and suppression of tails generated by pedestrian-inherent Doppler-frequency spreading.

Firstly, the values inside each row of the sliding reference window in the RDM are processed by an ordered statistic CFAR (OS-CFAR). This suppresses Doppler extensions without masking multi-target situations. The $k^{t h}$ ordered value is selected for each row of the RDM. In a second step, cell averaging CFAR (CA-CFAR) estimates the noise power level $\mu$ as the arithmetic mean of the values selected by the OS-CFAR. Thus, this technique is called OSCA-CFAR [6]. The decision threshold is calculated as $T=\alpha \cdot \mu$, being $\alpha$ a threshold factor that controls the false alarm rate in accordance to the NeymanPearson criterion. The decision test compares this threshold $T$ with the CUT value and chooses whether it is a target or not. Its output is shown in Fig. 4.

\section{B. Interpolation}

OSCA-CFAR application achieves an index list. Frequencies of the targets can be evaluated from their indexes in the discrete 2D spectrum stored in the RDM with a resolution depending on the samples of the signal. However, this resolution can be improved without raising sampling frequency of the signal, which is best to keep as low as possible, in order to maintain low hardware complexity. In order to do so, a discrete spectrum interpolation is applied to the indexes in the RDM [7]. Let $S_{d}[k]$ be a discrete magnitude spectrum, where a local maximum can be found at index $k_{m}$. A Gaussian curve $S_{c}(\varphi)$,

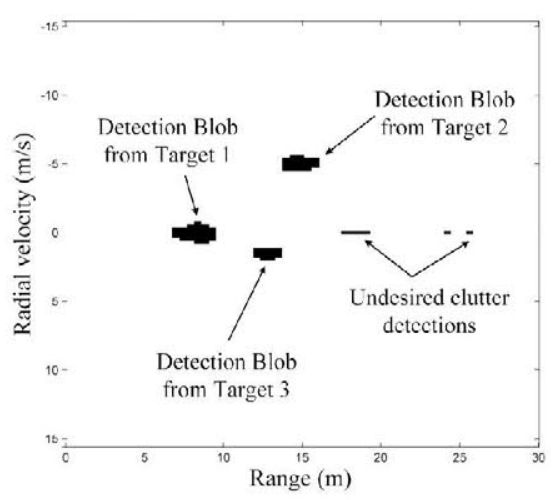

Fig. 4. OSCA-CFAR result for RDM example in Fig. 3. Not only targets have been detected, but also ground reflection. Therefore, a tracking stage is necessary to filter these detections that would generate false alarms. 
where $\varphi$ is the continuous counterpart of $k$, can be fit between the magnitude values $S_{d}\left[k_{m}-1\right], S_{d}\left[k_{m}\right]$ and $S_{d}\left[k_{m}+1\right]$ of three consecutive bins. Then, it is possible to find the abscissa $\varphi_{m}$ of the interpolated Gaussian curve maximum. This method allows a huge accuracy with a sampling frequency not higher that the one that fulfills Nyquist-Shannon theorem [8]. Having improved frequency measurements in both dimensions, targets ranges and radial velocities are computed and used as input for the data processing stage.

\section{DATA PROCESSING}

The double-tracking architecture explained in section I-B performs two crucial tasks:

1) Target tracking. To fulfill very-low-FAR requirement, the processing chain applies a double-tracking architecture. Tracking methods help keeping only persistent detections such as generated by real targets, and deleting the ones caused by variable clutter and noise.

2) Data fusion. Thanks to overlapping radiation patterns of the sensor network, position of the targets can be obtained using quadratic position-fixing algorithms. This operation demands a lot of computational cost and can lead to ghost targets in multi-target situations, so several methods are weighed to get the best results.

\section{A. Target tracking}

Target tracking is an essential requirement for warning systems employing a sensor network to understand the environment. Radar sensors report detections not only from targets of interest but also form other sources such as clutter. Therefore, target tracking associates sensor data from one cycle to stored detections from previous cycles to keep only persistent detections. As shown in Fig. 5, both stages perform three basic steps:

1) Plot-to-track association. The goal of an association process is to select all measured ranges for a single target. In a multi-target situation, there is an ambiguity in the possible combinations of measured ranges which has to be solved. To determine the most likely combination, association is performed using gating techniques and global nearest neighbor (GNN) approach [9] through the resolution of an assignment problem using Munkres algorithm [10].

2) Track update. Tracks are refreshed with associated plots, not assigned plots are stored as new tentative tracks, and not assigned tracks are annotated as such. When a track is not assigned for a certain number of cycles, it is deleted.

3) Prediction and filtering. Having speed (radial velocity or inter-cycle position change) through several cycles and other environment information makes the system able to predict, on the basis of its stored track data, the expected position for the target in the next cycle. These predictions are calculated using a basic $\alpha-\beta$ filter and will be used in next detection plot-totrack association. In addition, usage of both predicted and measured information allows to generate a more continous output data.

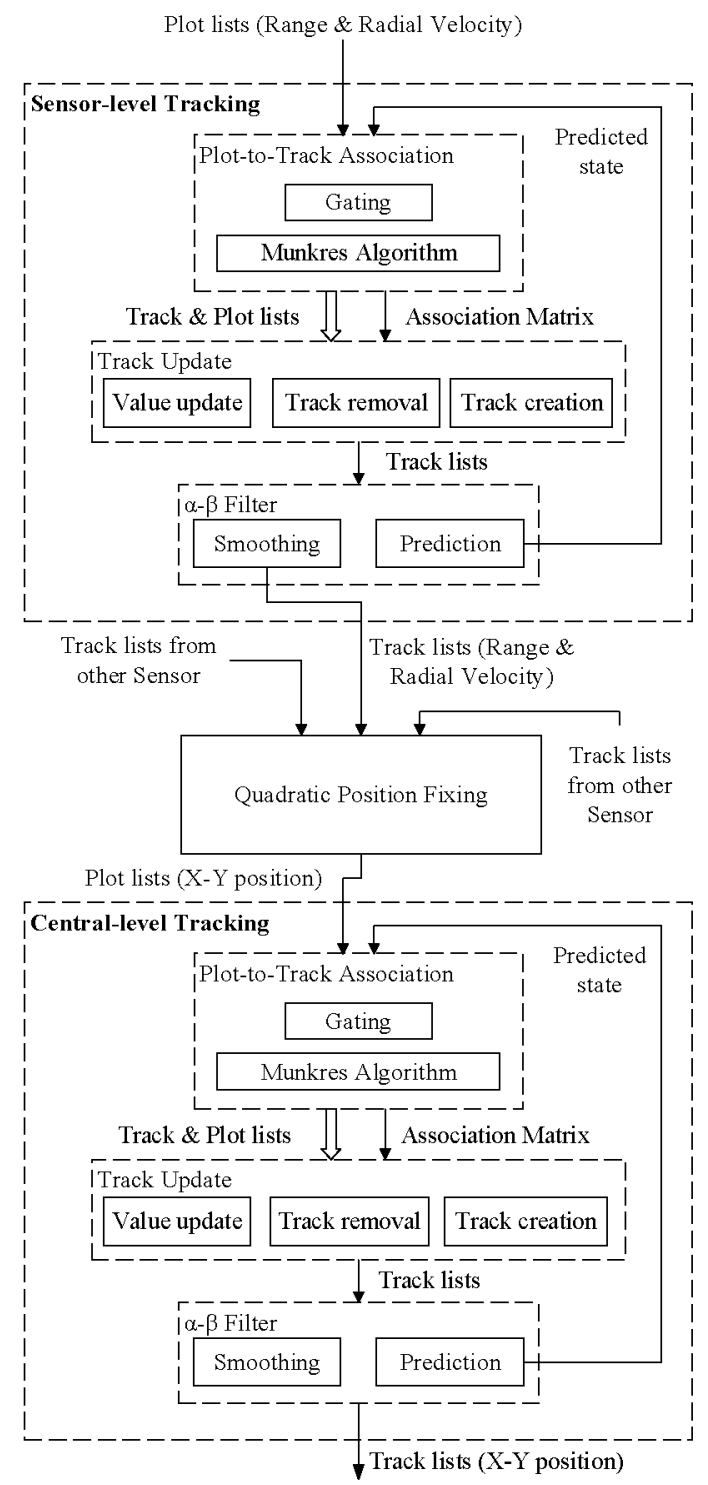

Fig. 5. Tracking scheme at both sensor and central level.

After the application of these three tasks, reliable and continuous datasets are obtained. This data is used as input for the quadratic position-fixing in the case of sensor-level tracking and for the alarms display in the case of central-level tracking. An example of the latest can be seen in Fig. 6.

\section{B. Data fusion}

With at least two measured ranges $r_{i}$, a target's position $t$ can be calculated as the intersection of two circles around the two sensors with the radius being the measured range. This process is called quadratic position-fixing algorithm. However, every sensor includes a range error. Because of that, real acquired measurements $\hat{r}_{i}$ are superimposed by error terms. These errors cause ambiguities that must be solved for leastsum-squared-error. Minimization of sum-squared-error means to find the target position $\hat{t}$ that best fits to all the measured ranges $\hat{r}_{i}$. To compute $\hat{t}$, a nonlinear optimization procedure, such as the iterative Gauss-Newton algorithm, has to be performed. 


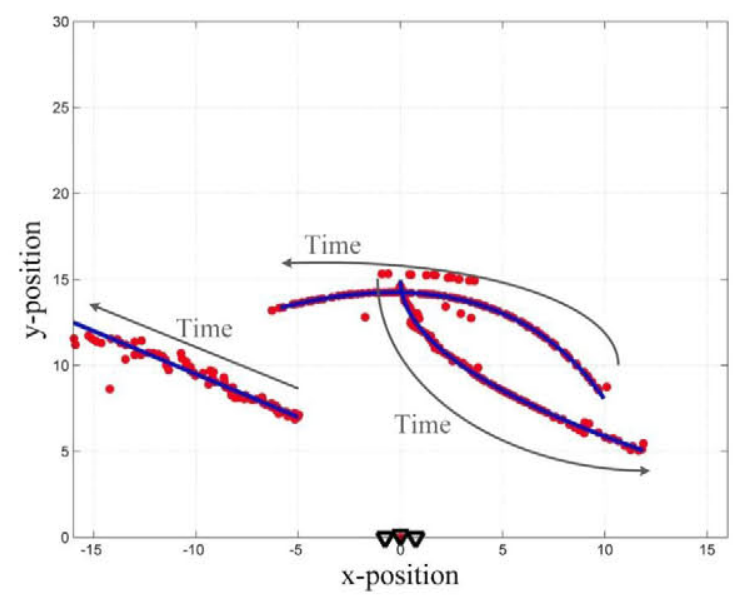

Fig. 6. Central-level tracking result example. Trajectory of targets are in blue, position estimations after central-level tracking are in red. It is clear to see correct performance of the system. It can happen tough, that a track is lost during target's movement, as can be seen in the leftmost target (gaps between position estimations), but soon after a new track replaces it.

In multi-target situations, every sensor $i$ will provide a set $R_{i}=\left\{\hat{r}_{i 1}, \ldots, \hat{r}_{i k_{i}}\right\}$ of $k_{i}$ measured ranges, so a preliminary data-association process has to be executed. Its objective is to select all measured ranges $\hat{r}_{i j}$ from each sensor $i$ corresponding for the same single target $j$. The combination of measured ranges that do not belong together will lead to ghost-target situations at the output of the quadratic position-fixing stage. Classical way to solve position-estimation problem consisting in data-association and quadratic position-fixing algorithm (usually called the top-down approach) can carry a technical challenge in dense multi-target situations [11]. Because of this, an alternative position-estimation process is chosen for the proposed system.

The alternative algorithm is based on a finite set of possible target positions inside the observation area. For an assumed position $\hat{t}$ taken as a reference, optimal data association can be performed based on a simple minimum-distance calculation. This approach is referred as bottom-up processing [11]. To obtain reference points for assumed target positions, a grid is used over the entire observation area as shown in Fig. 7. All individual grid points $\hat{t}$, located at a distance $r_{i}(\hat{t})$ from sensor $i$, are treated as possible target positions.

The minimal estimation error $E(\hat{t})$ at the considered grid point $\hat{t}$ is given by the sum of the minimal single-sensor distances between the predefined geometrical distance $r_{i}(\hat{t})$ to sensor $i$ and the best fitting measured range $\hat{r}_{i}$. Error value $E(\hat{t})$ defines a discrete function over the two-dimensional observation area. Final target positions are located at local minima of this function.

\section{CONCLUSION}

This paper has presented a system description of a radar network based on recently developed high-range resolution technology at K-Band. This system proves better that current employed methods. Despite its feasibility, several technical issues arise from complexity of the network, so a processing chain is designed and implemented. An implementation of

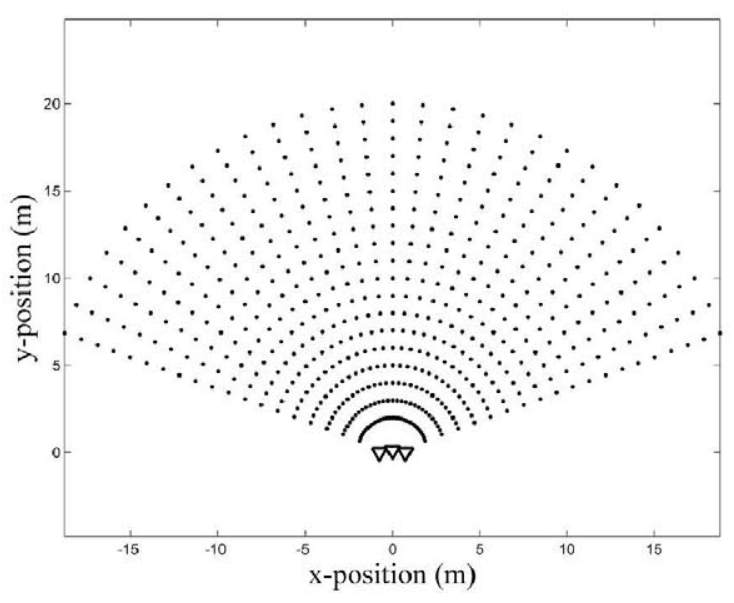

Fig. 7. Grid used in bottom-up processing. For every point in the grid $\hat{t}$, the error obtained if estimating the position in that point $E(\hat{t})$ is evaluated. Local minima will be the most suitable (least-sum-error) position.

such a radar network is a highlight of this work, because practical experiences are worth much more than only theoretical evaluation of system feasibility.

\section{ACKNOWLEDGMENT}

This work has been supported by the project TEC201128683-C02-01 of the Spanish National Board of Scientific and Technology Research.

\section{REFERENCES}

[1] M. Klotz, "An Automotive Short Range High Resolution Pulse Radar Network," Ph.D. dissertation, Technischen Universität HamburgHarburg, Hamburg, Germany, 2002.

[2] R. Poisel, Electronic Warfare Target Location Methods. London, UK: Artech House, 2005.

[3] J. T. González-Partida, F. León-Infante, R. Bláquez-García, and M. Burgos-García, "On the Use of Low-Cost Radar Networks for Collision Warning Systems Aboard Dumpers," Sensors, no. 14, pp. 3921-3938, 2014.

[4] S. Blackman and R. Popoli, Design and Analysis of Modern Tracking Systems. London, UK: Artech House, 1998.

[5] H. Rohling, "Radar CFAR Thresholding in Clutter and Multiple Target Situations," IEEE Trans. Aerosp. Electron. Syst., vol. 19, pp. 608-621, Jul. 1983.

[6] M. Kronague and H. Rohling, "Fast Two-Dimensional CFAR Procedure," IEEE Trans. Aerosp. Electron. Syst., vol. 49, no. 3, pp. 1817 1823, Jul. 2013.

[7] J. L. Gonzalez and M. Gasior, "Improving FFT Frequency Measurement Resolution by Parabolic and Gaussian Interpolation," 2004, pp. 276285.

[8] A. V. Oppenheim and R. W. Schafer, Discrete-Time Signal Processing. Engewood Cliffs, NJ: Prentice Hall, 1989.

[9] P. Konstantinova, A. Udvarev, and T. Semerdjiev, “A Study of a Target Tracking Algorithm Using Global Nearest Neighbor Approach," in CompSysTech'03 International Conference on Computer Systems and Technologies, 2003, pp. 290-295.

[10] F. Bourgeois and J.-C. Lassalle, "An Extension of the Munkres Algorithm for the Assignment Problem to Rectangular Matrices," in Communications of the ACM, no. 12, Dec. 1971, pp. 802-804.

[11] F. Fölster and H. Rohling, "Data Association and Tracking for Automotive Radar Networks," IEEE Trans. Intell. Transp. Syst., vol. 6, no. 4, pp. 370-377, Dec. 2005. 